\section{GATA3 IMMUNOHISTOCHEMICAL STAIN IS A HIGHLY SENSITIVE TOOL FOR DISTINGUISHING BETWEEN UPPER UROTHELIAL TRACT UROTHELIAL CARCINOMA AND LUNG SQUAMOUS CELL CARCINOMA AND ADENOCARCINOMA}

J. du Plessis $^{1}$, M. Newman ${ }^{2}$, D. Williams ${ }^{2}$

${ }^{1}$ Department of Anatomical Pathology, Healthscope Pathology, Clayton, and ${ }^{2}$ Department of Anatomical Pathology, Austin

Pathology, Austin Hospital, Heidelberg, Vic, Australia

\begin{abstract}
Aim: With recognition of the overlapping diagnostic features between metastatic upper urothelial tract urothelial carcinoma (UUT-UC) and primary lung squamous cell carcinoma (SCC) and adenocarcinoma, this study examined the expression profile of GATA3 and Uroplakin III (UP) in these tumours.

Method: Immunohistochemical staining for GATA3 and UP was performed on tissue microarrays from 55 UUT-UCs and 32 primary lung cancers (13 adenocarcinomas and 19 SCCs).

Results: 52 of UUT-UCs labelled GATA3 positive (94.5\%), with 1 graded as $1+$ staining $(5-25 \%$ of nuclei) with all remaining positive cases graded as 3+/4+ staining (viz. 50-75\%/ $>75 \%$ of nuclei labelling). A lower proportion of UUT-UCs were UP positive (19 cases, 34.5\%). Zero lung carcinomas were positive for UP, and 2 SCCs exhibited 1+ marking for GATA3 (10\%) with no adenocarcinomas staining.

Discussion: Metastatic UUT-UC is recognised to engender diagnostic difficulty when encountered in small lung biopsy, with squamoid morphology and occasional focal squamous and/ or glandular differentiation. Added complexity is the overlapping immunophenotype of p63 and/or CK5/6 positivity with SCC, and the usual anamnesis of cigarette smoking in both patient populations. This study demonstrates that GATA3 IHC is highly sensitive for UUT-UC and has the potential to provide a powerful adjunct to historical diagnostic tools.
\end{abstract}

\section{TANYCYTIC EPENDYMOMA VS PILOCYTIC ASTROCYTOMA? PITFALLS IN DIAGNOSIS}

\author{
J.M. Dyke $^{1}$, R.C. Junckerstoff ${ }^{1,2}$, V.A. Fabian ${ }^{1,2}$ \\ ${ }^{1}$ Neuropathology, PathWest, Royal Perth Hospital, and ${ }^{2}$ School \\ of Pathology and Laboratory Medicine, University of Western \\ Australia, WA, Australia
}

Case report: Two cases are presented, a T5/6 intramedullary spinal cord tumour and a right cerebellopontine angle (CPA) tumour. At the time of intraoperative assessment, smears from both tumours showed atypical spindled cells with fibrillary cytoplasm, the differential diagnosis including pilocytic astrocytoma or tanycytic ependymoma. Light microscopy and immunohistochemical staining did not definitively differentiate between these two diagnoses. Electron microscopy confirmed the ependymal nature of the neoplastic cells, with the CPA tumour being reclassified as a tanycytic ependymoma after initially being reported as a pilocytic astrocytoma.

Discussion: Tanycytic ependymoma was first described in 1978 by Friede and Pollak. ${ }^{1}$ Along with pilocytic and pilomyxoid astrocytomas, these share radial glial cells (tanycytes) as the putative cell of origin. ${ }^{2}$ Cytomorphology and immunophenotype of these tumours show much overlap. These lesions can be differentiated on ultrastructural examination. The distinction between these entities is important as pilocytic astrocytomas are
WHO grade I neoplasms, whereas tanycytic ependymomas and pilomyxoid astrocytomas are WHO grade II. Molecular pathology may also help with classification.

\section{References}

1. Friede RL, Pollak A. The cytogenetic basis for classifying ependymomas. J Neuropathol Exp Neurol 1978; 27: 103-18.

2. Lehman NL. Central nervous system tumours with ependymal features: A broadened spectrum of primarily ependymal differentiation? J Neuropathol Exp Neurol 2008; 67: 117-88.

\section{USE OF CLINICAL AND MORPHOLOGICAL INDICATORS IN DIAGNOSING MICROSATELLITE INSTABILITY IN ENDOMETRIOID CARCINOMA}

\section{Harini Edirisinghe, Anita Mani \\ Pathology North, Tamworth Base Hospital, Tamworth, NSW, Australia}

Background: Endometrioid carcinoma is the most common non-colorectal carcinoma to occur in Lynch syndrome, which causes mutations in the DNA mismatch repair gene, resulting in microsatellite instability (MSI). There are specific clinical and morphological indicators of MSI. Having a high degree of suspicion is important in diagnosis of Lynch syndrome.

Case report: A 43-year-old female with history of uterine fibroids underwent routine hysterectomy with bilateral salphingooophorectomy. The entire uterus was involved by a grade II endometrioid carcinoma with patchy areas of high grade nuclear features. The tumour was staged as FIGO stage IB. Due to the young age of the patient, with no apparent history of unopposed oestrogen stimulation, and the relatively high grade, extensive disease, the possibility of MSI related tumour was considered. Immunohistochemistry showed lack of staining in MLH1 and PMS2, which suggests that this tumour is a microsatellite unstable tumour. The patient was then referred for genetic counselling and further testing for definitive diagnosis of Lynch syndrome.

Discussion: Consideration of possible MSI especially in situations where a tumour does not develop in the background of common risk factors is important in the diagnosis of Lynch syndrome.

\section{CASE REPORT: MALE BREAST CARCINOMA WITH NEUROENDOCRINE DIFFERENTIATION}

Harini Edirisinghe, Joseph Lee, Anita Mani

Pathology North, Tamworth Base Hospital, Tamworth, NSW, Australia

Background: Carcinoma of the male breast is a rare, but distinctly recognisable entity. It accounts for $<1 \%$ of all breast cancers. Neuroendocrine differentiation is a rare morphological pattern seen in breast carcinoma. Therefore carcinoma of the male breast with neuroendocrine differentiation is an even rarer entity.

Case report: An 81-year-old male with no previous history of cancer presented with a left sided axillary lymphadenopathy. Core biopsy of the lesion showed a poorly differentiated large cell malignancy with an immune-profile consistent with a neuroendocrine tumour.

He went on to have left sided mastectomy with axillary clearance, which showed a well-circumscribed nodular tumour within 